\title{
Liquid crystal television (LCTV) as an optics education tool
}

\section{Michael Giles, Narasimha Prasad, Sean Doyle}

Michael K. Giles, Narasimha S. Prasad, Sean M. Doyle, "Liquid crystal television (LCTV) as an optics education tool," Proc. SPIE 2525, 1995 International Conference on Education in Optics, (13 October 1995); doi: $10.1117 / 12.224058$

SPIE Event: SPIE's 1995 International Symposium on Optical Science, Engineering, and Instrumentation, 1995, San Diego, CA, United States 


\title{
The Liquid Crystal Television (LCTV) as an Optics Education Tool
}

\author{
Michael K. Giles, Narasimha S. Prasad, and Sean M. Doyle \\ The Klipsch Department of Electrical and Computer Engineering \\ Box 30001, Dept. 3-0 \\ New Mexico State University \\ Las Cruces, NM 88003-0001
}

Tel: (505)646-3115, FAX:(505)646-1435

email:mgiles@nmsu.edu

\begin{abstract}
In this paper, it is shown that twisted nematic liquid crystal television display panels (LCTV's) can be used as programmable optical elements to demonstrate basic optical phenomena (e.g., interference and diffraction) in the student optics laboratory. The LCTV's considered are pixelated with gray levels appropriately manipulated to generate an optical element by electrically addressing the LCTV using image processing software. Programmable optical elements such as single or multiple apertures and slits, gratings (amplitude and phase), apertures with programmable shape and size, phase objects (wavefronts), and programmable pupil functions such as anamorphic lenses, lenslet arrays, and spherical lenses with programmable size, focal length, and generalized pupil functions that include wavefront aberration functions are discussed and the methods used to generate them are described.. It is demonstrated that the LCTV combines the merits of discrete optical elements and graphic user interface based software tools to provide a simple and inexpensive means to study optical elements.
\end{abstract}

\section{INTRODUCTION}

Optics education is becoming an essential discipline required for students working in various fields because of the importance of a wide variety of optical techniques and instruments. Optical instruments consist of one or more optical elements such as mirrors, prisms, gratings, lenses, apertures, etc., that are either passive or active. These elements manipulate either conventional or laser radiation via the basic optical phenomena of reflection, refraction, diffraction, and interference. A study of optical elements forms the core of optics education. Using mathematical formulations, the behavior of optical elements can be studied. However, for many beginners in optics, understanding optics directly through equations is a difficult and slow process. To facilitate quick grasping of underlying conceptual schemes, commercial or custom designed. hardware and software tools are available. Although traditional discrete optical elements are used to demonstrate elegantly the respective optical phenomena, they are of fixed specifications so that the study of optical behavior for various specifications with such elements is cumbersome and expensive. Software tools based on mathematical formulations and graphical user interfaces that are available to predict the behavior of optical elements cannot provide the intuitionbuilding multisensory learning experience of a visual demonstration. In this context, we propose an alternative optics education tool using an LCTV which can be programmed to behave as a desired optical 
element whose specifications can be continuously varied. This tool is interactive through a computer and is shown to provide visual demonstrations expeditiously in real time and yet is simple and inexpensive. It is shown that the LCTV-based optics tool combines the advantages of both hardware and software techniques and hence can be considered to be a more effective tool than those available at present.

The programmable optical elements that are considered in this paper include apertures of different sizes and shapes for the study of diffraction, multiple apertures, spherical lenses with generalized programmable pupils for the study of lens aberrations and diffraction, anamorphic lenses, and lenslet arrays. In addition, this tool has been used to generate amplitude and phase gratings ${ }^{1}$, to deflect a laser beam $^{2}$, and to sense and correct a optical wavefronts when used in conjunction with an interferometer ${ }^{3}$. In addition, the LCTV has been used extensively as an input and/or filter transducer in optical correlator systems. ${ }^{4}$ In section 2 the basic properties of an LCTV, the ease with which it is programmed, and its modes of operation are described. Examples of programmable elements and their output characteristics are presented in section 3. Conclusions are presented in section 4.

\section{THE LCTV AND ITS MODES OF OPERATION}

The Epson liquid crystal television, obtained from an Epson Crystal Image Video Projector model 1020, was used. The projector contains three thin film transistor LCTV's. Each LCTV has a 1.3 inch diagonal screen. This screen contains an array of 320 X 220 rectangular pixels, each having dimensions of $60 \times 70$ micrometers with a pitch of $80 \times 90$ micrometers as shown in Figure 1.

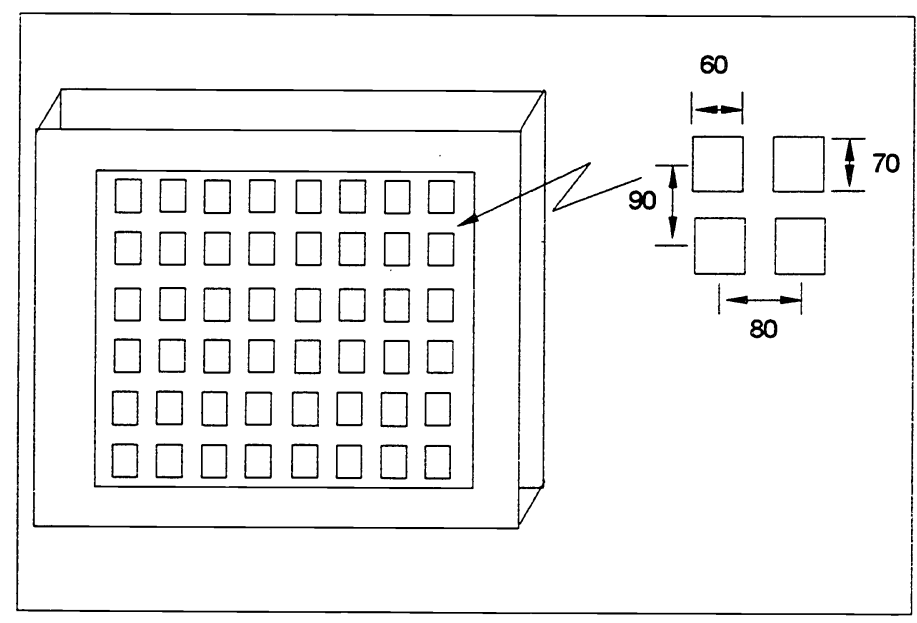

Figure 1. The LCTV pixel configuration. The units are in micrometers.

Each pixel consists of a twisted nematic liquid crystal sandwiched between two glass plates. The polarization of the incident light will follow the orientation of the liquid crystal molecules as it propagates through it and hence twisted nematic liquid crystals serve as polarization rotators. The brightness control can be adjusted to provide a bias voltage across the entire array of pixels, while the voltage across each individual pixel is modulated by the video signal received by the projector. The applied electric field 
across the pixel alters the twist and also the tilt of the molecules with respect to the director. The tilting effect modulates the extraordinary refractive index and hence changes the rotation of the polarization of the propagating light. Since the amount of rotation depends on the applied voltage across the pixel, a LCTV placed in between crossed polarizers produces a gray scale picture. The LCTV can be operated as a continuous amplitude or phase modulator depending upon the applied voltage. Amplitude modulation occurs at high bias voltages whereas phase modulation occurs at low bias voltages. In the off-state ( no applied external field) the amount of polarization rotation (rotary power) of our Epson device is about $70^{\circ}$. The LCTV provides a throughput of $75 \%$. The theory of twisted nematic liquid crystals has been discussed extensively in the literature. ${ }^{5,6,7}$

\subsection{Programming the LCTV}

The Epson video projector is equipped with three audio/visual inputs, one BNC type connector for each liquid crystal light valve. The Epson device also has a digital RGB input. The TTL signal from an IBM PC clone in CGA low resolution mode can be sent directly to the video projector. However, the CGA mode of operation has a lower resolution than the analog RGB video mode of operation. An IBM PC is used with an image processing board that sends a composite NTSC (3.8 $\mathrm{MHz})$ encoded video signal to the video projector. ${ }^{9}$

Twisted nematic liquid crystals are uniaxial with appreciable birefringence. Hence they possess two eigen-polarization states which can be determined by placing them between two polarizers. The Epson LCTV's are fabricated such that their eigen-polarization states are along vertical and horizontal directions. These eigen-polarization states correspond to the ordinary and extraordinary axes of the twisted nematic liquid crystal. The extraordinary axis in the elongated direction of the molecules is called the director. Polarization components of the incident light waves that are aligned with the director at the LCTV input face are modulated in phase. Thus the director can be located by using phase measuring schemes. For example, by using an interferometer and observing the fringe shift produced for a given bias voltage across the liquid crystal, the director can be located because the maximum fringe shift occurs when the polarization of the incident light is aligned with the director. The phase modulation results when the retardance is modulated with the applied voltage. To avoid phase modulation, the incident light should be polarized orthogonal to the director.

The polarization rotation capability enables the LCTV to be used in an amplitude mode, binary phase mode, and an amplitude coupled with binary phase mode. For programming convenience, the system presented in this paper used only the central $128 \times 128$ pixels of the Epson device. Using the $\mathrm{C}$ programming language code, any selected pixel or group of pixels was addressed to present a gray level value between 0 and 255 to the LCTV operating in several different modes below.

\subsubsection{Amplitude Mode}

The LCTV is placed between two polarizers. The input polarizer is aligned with one of the eigen polarization states. The ordinary axis which has very little phase modulation associated with it is best. The second polarizer is oriented orthogonal to the rotated output polarization state (the twisted angle of 
the device) produced by gray level 0 . This is the mode used to generate simple transmissive apertures of different sizes and shapes, multiple apertures, and amplitude gratings.

\subsubsection{Binary Phase Mode}

Again, the LCTV is sandwiched between two polarizers. The input polarization is oriented orthogonal to the director at the front surface, and the output polarizer is oriented orthogonal to the bisector of the poarization directions for the applied voltage levels associated with gray levels 0 and 255 . Only two gray levels are used to drive the LCTV, usually 0 and 255, each producing equal amplitude transmittance through the system but with opposite phases $\left(0\right.$ and $\left.180^{\circ}\right)$. Binary phase gratings and binary phase Fresnel lenses are implemented using this mode.

\subsubsection{Amplitude Coupled with Binary Phase Mode ${ }^{9}$}

In this mode, the LCTV acts as a real function generator (positive and negative real values). It is identical to the binary phase mode, but all gray level values are used so that the binary phase outputs have different amplitudes, each depending on the gray level with which it is driven. Any real function can be implemented using this mode, including certain lens functions.

\subsubsection{Phase Mostly Mode}

For phase modulation, the input polarizer is aligned with the director and no output polarizer is needed. This is the mode used to generate the lenses with generalized pupil functions presented in this paper. Because the polarization at the output will vary somewhat for pixels driven with a variety of gray levels, an output polarizer placed approximately at the mean direction of all of the output polarization components can be used to reduce background somewhat and thus increase the contrast of the patterns generated by the system.

\section{PROGRAMMABLE OPTICAL ELEMENTS}

Figure 2 shows the experimental setup used to demonstrate the capabilities of the programmable LCTV used to implement a variety of programmable pupil functions. Two types of pupils were realized-simple transmissive apertures including multiple apertures of various shapes and sizes and continuous phase Fresnel lenses ${ }^{2}$ of different focal lengths.

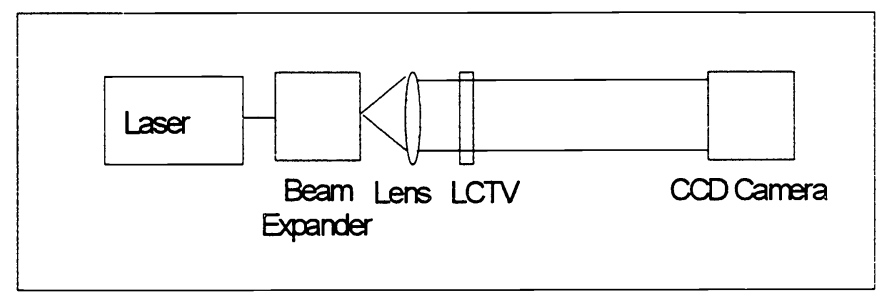

Figure 2. Experimental set-up. 
Simple apertures generated using the binary phase mode can be used to teach basic diffraction theory. Figure 3 contains five examples of apertures and their corresponding diffraction patterns generated with this system--a large aperture, a small aperture, two small separated apertures to demonstrate Young's experiment, a vertical elliptical aperture and a horizontal elliptical aperture. A lens is placed in the collimated beam just after the LCTV in Figure 2, and the CCD camera is placed a focal length away from the lens in order to study the diffraction patterns produced by the various apertures. The study of diffraction is facilitated by the ease with which the aperture size and shape can be changed with a user-friendly aperture generating program.

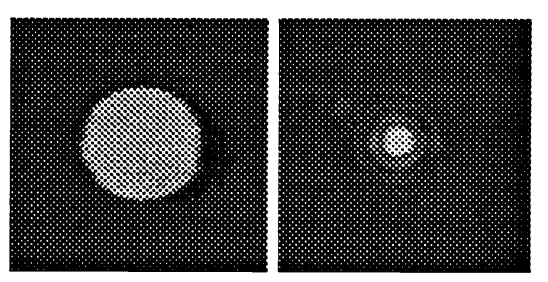

(a)

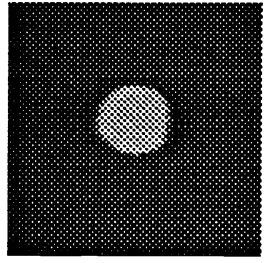

(b)

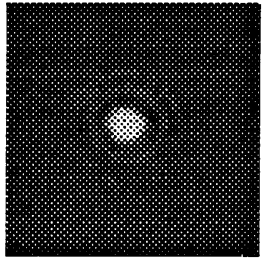

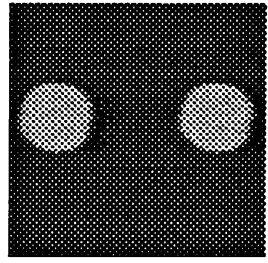

(c)

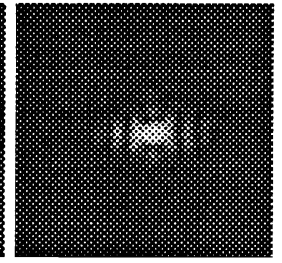

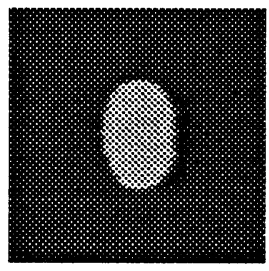

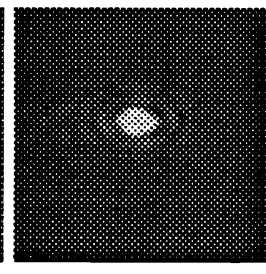

(d)
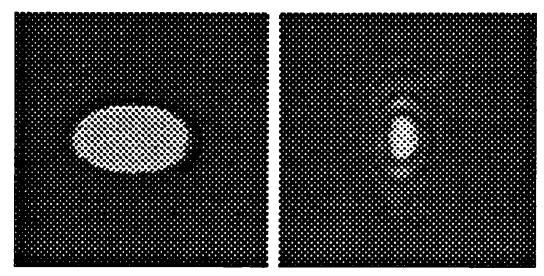

(e)

Figure 3. Diffraction patterns obtained by writing programmable apertures on the LCTV: (a) circular aperture, (b) smaller circular aperture, (c) two separated circular apertures, (d), (e) elliptical apertures.

Figure 4 presents examples of programmable pupils using phase Fresnel lenses and their resulting point spread functions. These point spread functions are generated directly using the set-up of Figure 2 as shown and placing the CCD camera at the selected focal length of the phase Fresnel lens. Figures $4 \mathrm{a}$ and $4 \mathrm{~b}$ show a simple Fresnel lens having a focal length of $50 \mathrm{~cm}$ and the same lens with approximately one wave of 3 rd order coma together with their respective point spread functions. Figures $4 \mathrm{c}$ through $4 \mathrm{f}$ show the point spread functions obtained by programming the lens pupil of Figure 4a with approximately two waves of coma, approximately one wave of astigmatism (tangential focus), approximately two waves of balanced astigmatism (medial focus), and approximately two waves of balanced spherical aberration (near the best geometrical focus). Generalized pupil functions ${ }^{10}$ are easy to generate using this system since the continuous wavefront aberration polynomial phase function can be added directly to the phase of the Fresnel lens function and written directly to the LCTV. Accurate calibration of the system can be accomplished by placing the programmable pupil in one leg of an interferometer and analyzing the resulting fringe patterns. 


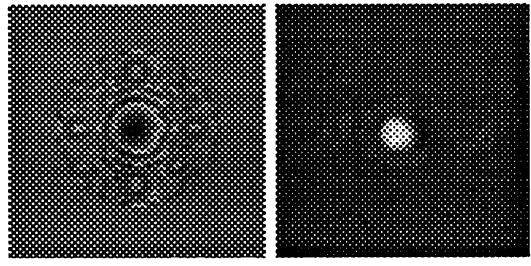

(a)

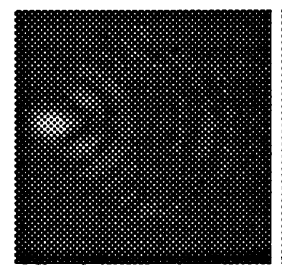

(c)

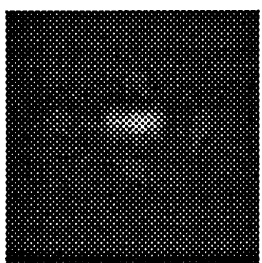

(d)
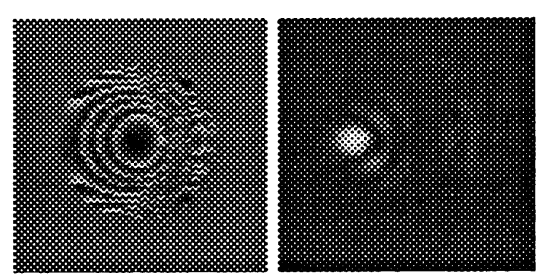

(b)

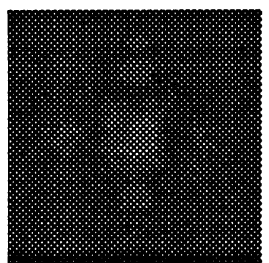

(e)

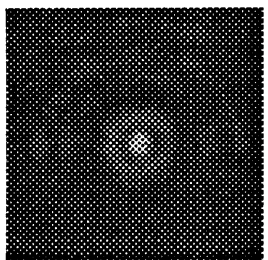

(f)

Figure 4. Generalized pupils and their diffraction point spread functions (PSF) obtained by writing the pupils on the LCTV: (a) phase Fresnel lens with a $50 \mathrm{~cm}$ focal length and its PSF, (b) the same lens with approximately one wave of coma and its PSF, (c) PSF from the phase Fresnel lens of (a) with approximately 2 waves of coma, (d) PSF from the lens of (a) with approximately 1 wave of astigmatism (tangential focus), (e) PSF from the lens of (a) with approximately 1 waves of astigmatism (medial focus), and ( $\mathrm{f}$ ) PSF from the lens of (a) with approximately 2 waves of balanced spherical aberration (near best focus).

Figure 5 illustrates the use of the programmable pupil (LCTV) to generate a simple anamorphic lens which produces a line focus and an array of lenslets, each of which produces a point image. Only four of the point images from the $4 \times 4$ array of lenslets are shown. Of course, the figures presented in this paper represent only a few of the myriad of programmable pupils that can be generated using the LCTV. Tilt, defocus, and other aberrations can also be implemented with the LCTV.

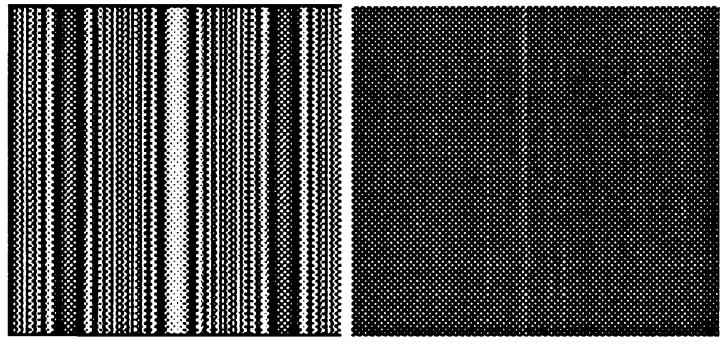

(a)

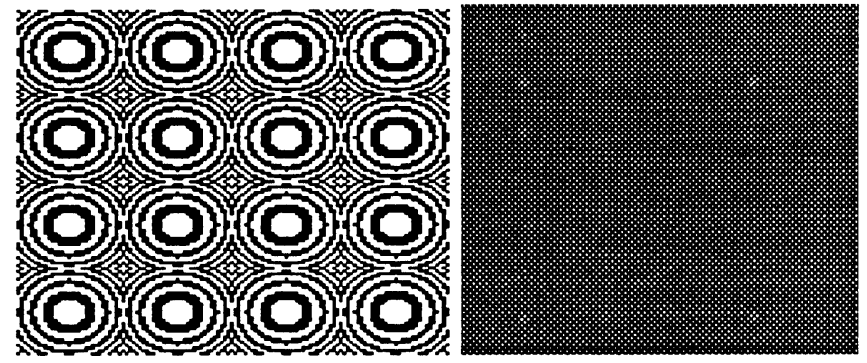

(b)

Figure 5. Additional LCTV pupil functions: (a) an anamorphic lens and its line focus, (b) a 4 X 4 array of lenslets and four of the sixteen point images it generates. 


\section{CONCLUSIONS}

Inexpensive twisted nematic LCTV's have been used successfully to demonstrate basic optical phenomena. In this paper the use of the LCTV as a programmable pupil has been demonstrated and the ease with which it can be used to generate apertures and generalized pupils for classroom demonstrations has been described. The use of the LCTV takes on another dimension in the classroom setting which cannot be adequately described in a paper such as this. That is the dimension of time. The LCTV can be programmed to generate any desired aperture or generalized pupil function and change the pupil function rapidly at a rate of 30 frames per second if desired. This makes it possible for the student to study the diffraction phenomenon as the pupil is changing and to assess the effects of subtle changes in the pupil function. For example, a given aberration function can be written to the LCTV, and the user can observe the effects on the PSF caused by changing the amount of defocus. Similarly, the student can observe the effects of changing the aperture size or the separation of two apertures in real time by controlling these parameters with the touch of a key or the movement of a mouse.

\section{REFERENCES}

1. R. Dou and M. K. Giles, SPIE Proceedings, Vol. 2566, July 1995.

2. N. S. Prasad, S. M. Doyle, and M. K. Giles, SPIE Proceedings, Vol. 2537, July 1995.

3. R. Dou and M. K. Giles, Optics Letters, vol. 20, pp. 1583-1585, 1995.

4. N. Clark, C. M. Crandall, and M. K. Giles, SPIE Proceedings, Vol. 1564, pp. 439-451, 1991.

5. L. M. Blinov, Electrical and Magneto-Optical Properties of Liquid Crystals, John Wiley \& Sons Ltd., New York, 1983.

6. A. Yariv and P. Yeh, Optical Waves in Crystals, John Wiley \& Sons, New York, 1984.

7. B.E.A. Saleh and M. C. Teich, Fundamentals of Photonics, John Wiley \& Sons, Inc., New York, 1991.

8. Epson Liquid Crystal Video Projector Service Manual, Epson Corp., 1991.

9. N. Clark, Design and Performance of Optical Correlator Systems Using LCTVs, Ph.D. Dissertation, New Mexico State University, 1993.

10. J. D. Gaskill, Linear Systems, Fourier Transforms, and Optics, John Wiley \& Sons, Inc., New York, p. $387,1978$. 